\title{
Atmospheric turbulence at the South Pole and its implications for astronomy
}

\author{
T. Travouillon ${ }^{1}$, M. C. B. Ashley ${ }^{1}$, M. G. Burton ${ }^{1}$, J. W. V. Storey ${ }^{1}$, and R. F. Loewenstein ${ }^{2}$ \\ 1 School of Physics, University of New South Wales, Sydney, NSW 2052, Australia \\ 2 University of Chicago, Yerkes Observatory, 373 W. Geneva Street, Williams Bay, WI 53191, USA
}

Received 12 August 2002 / Accepted 6 December 2002

\begin{abstract}
To investigate the low-atmosphere turbulence at the South Pole, we have measured, using a SODAR, the temperature fluctuation constant $\left(C_{T}^{2}\right)$ during winter, as a function of altitude up to $890 \mathrm{~m}$. We found that the turbulence was on average concentrated inside a boundary layer sitting below $270 \mathrm{~m}$. While at the peak of winter the turbulence was stable and clearly bounded, during other seasons there was a more complex turbulence profile which extended to higher altitudes. We found that this behaviour could be explained by the horizontal wind speed conditions whose altitude profile closely matched the turbulence profile. We also observed the presence of a vertical wind velocity change of direction at an altitude range corresponding to the turbulent region. The turbulence gives rise to an average seeing of $1.73^{\prime \prime}$, which compares poorly with the best astronomy sites. The location of the turbulence, however, means that the seeing quickly decreases above the boundary layer (dropping to $0.37^{\prime \prime}$ above $300 \mathrm{~m})$. We also have recorded the largest isoplanatic angle $\left(\theta_{\mathrm{AO}}=3.3^{\prime \prime}\right)$ and the longest coherence time $\left(\tau_{\mathrm{AO}}=2.9 \mathrm{~ms}\right)$ of any ground-based site.
\end{abstract}

Key words. site testing - turbulence - atmospheric effects - instrumentation: miscellaneous

\section{Introduction}

The unique climatic conditions offered by the Antarctic continent have encouraged astronomers to investigate its potential as an observatory location. In order to assess the quality of the sky in Antarctica, the AASTO program (Automated Astrophysical Site Testing Observatory) (Storey et al. 1996; Storey 1998) was initiated. The AASTO is a remote, autonomous observatory fitted with a variety of site testing experiments including near and mid infrared sky monitors, a fibre optic spectrometer, a submillimeter tipper and a SODAR (SOund Detection And Ranging). The infrastructure present at the South Pole made it the obvious first candidate site in a campaign aimed to eventually cover most of the high points of the Antarctic plateau including Dome C, Dome A and Vostok.

In this paper, we present SODAR measurements of the lower-atmosphere turbulence throughout the year 2000 . Astronomical seeing is a consequence of the path of light being distorted as it passes through turbulent cells of atmosphere with different temperatures and therefore different refractive indices. Zones of convection between hot and cold fronts create continuous large temperature gradients. In temperate sites (where most telescopes are located), a major contribution to the seeing comes from the two turbulence layers located in the troposphere. In Antarctica however, the type of turbulence

Send offprint requests to: T. Travouillon, e-mail: tonyt@phys.unsw.edu.au encountered is different. The latitude forbids the presence of a jet stream and any sort of high altitude convection zone. This was confirmed by the turbulence profiles recorded by Marks et al. (1999). Instead, a low altitude katabatic wind originating from the highest part of the Antarctic plateau is driven radially towards the coast of the continent, aided by the smooth slope and absence of obstacles. The cold ice covering the ground creates, in winter time, a temperature inversion a few hundred metres high within which most of the turbulence is confined. Previous turbulence studies (Marks et al. 1996, 1999) using a tower and balloon-launched microthermal measurements showed that $80 \%$ of the seeing originates within this boundary layer. With the SODAR, we have studied the turbulence profile of the lowest $890 \mathrm{~m}$ of the atmosphere in a continuous manner over a 10 month period to extend our knowledge of the seeing condition over the whole "night". The SODAR is capable of measuring the echo strength (which is proportional to the temperature fluctuation constant $C_{T}^{2}$ ), the horizontal and vertical wind speed, and wind direction. This information can also be used to derive parameters such as the seeing, the isoplanatic angle and the coherence time.

\section{Theory}

\subsection{Turbulence theory}

In this chapter, we assume that the atmospheric turbulence follows Kolmogorov's power law (Tatarski 1961). The mean 
squared difference of the temperature at two points separated by a distance $\rho$ is defined as:

$D_{T}(\rho)=<(\theta(r+\rho)-\theta(r))^{2}>$

where $\theta=T-<T>$ is the deviation of the temperature at a particular point from the average temperature. We assume that the structure function can only depend on the separation between the two points $\rho$, the rate of turbulent energy transfer $\varepsilon_{0}$ and the rate at which the turbulent energy produces fluctuation, $\eta_{0}$. A dimensional analysis gives (Roddier 1981):

$D_{T}=\eta_{0} \varepsilon_{0}^{-1 / 3} \rho^{2 / 3}=C_{T}^{2} \rho^{2 / 3}$,

where we have introduced the temperature fluctuation constant $C_{T}^{2}$. This parameter is empirically associated with Kolmogorov's power spectrum of temperature fluctuation, $\phi_{T}$, which is given by:

$\phi_{T}(\kappa)=0.033 \times C_{T}^{2} \kappa^{-5 / 3}$,

where $\kappa$ is the wave number. The SODAR sends a pulsed sound wave into the atmosphere that is partially reflected when it encounters a turbulent layer. The difference in temperature at the layer boundary changes the speed of the wave such that the larger the temperature gradient, the larger the backscattered signal. It was shown by Danilov (1992) that for a monostatic SODAR (i.e., one measuring $180^{\circ}$ reflections) the differential scattering cross-section $\partial \sigma$ from a volume $V$ is given by:

$\partial \sigma=2 \pi \kappa^{4} V\left[\frac{T^{2}}{4} \phi_{T}(2 \kappa)\right] \partial \Omega$,

where $T$ is the temperature and $\partial \Omega$ is the sampled angular size. This equation can be combined with Eq. (3) to give:

$C_{T}^{2}=\frac{\sigma_{180} T^{2}}{4 \times 10^{-3} \kappa^{1 / 3}}$

where $\sigma_{180}$ is the effective backscattering cross section. The SODAR therefore gives us a value proportional to $C_{T}^{2}$ by calculating the ratio of received power to transmitted power after correcting for noise and $d^{4}$ attenuation, where $d$ is the distance to the scatterer.

\subsection{Seeing theory}

By itself, the temperature fluctuation constant is not enough to describe the effect of the seeing. We need to take one more step and define the refractive index fluctuation constant, $C_{N}^{2}$. The variation of the refractive index, $n=N-\langle N\rangle$, is proportional to both the temperature and humidity gradient. It is usually considered that the humidity contribution is negligible and is not used in the calculation of the refractive index fluctuation constant (Roddier 1981). This assumption fits the South Pole conditions particularly well since the absolute humidity is close to zero. We can write $n=A \theta$, where the value for the constant $A$ is given by the Gladstone's relation:

$A=80 \times 10^{-6} \frac{P(h)}{T^{2}}$, where $P$ is the pressure in millibar, and $T$ the temperature in Kelvin. We can relate this equation to the temperature fluctuation constant and find at any altitude $h$ :

$C_{N}^{2}(h)=80 \times 10^{-6}\left(\frac{P(h)}{T^{2}}\right)^{2} C_{T}^{2}$.

This equation relates the SODAR measurements to the effect of turbulence on light propagation throughout the atmosphere. The $C_{N}^{2}$ profile integrated over the whole atmosphere then gives the seeing, using the following relation (described in detail in Fried 1966):

$\varepsilon_{F W H M}=5.25 \lambda^{-1 / 5}\left(\int_{0}^{\infty} C_{N}^{2}(h) \mathrm{d} h\right)^{3 / 5}$,

where $\varepsilon_{F W H M}$ is the angular size in arcseconds of the full width at half maximum of the point spread function and $\lambda$ is the wavelength of the light.

\section{System description and data processing}

\subsection{The SODAR}

Many techniques can be used to measure the turbulence and seeing. We selected a SODAR (Hecquet \& Klaus 1989; Crescenti 1997) as its characteristics best fit the requirements of our experiment. Besides the advantage of having a customisable range and resolution, the SODAR presents three key advantages:

- It is ideal for an unmanned experiment. The software and hardware provided make it totally remotely controllable. The SODAR's computer can be accessed remotely through satellite connection and allows direct control of the equipment. The data can be stored locally, yet can also be accessed remotely, therefore allowing for direct analysis and status check.

- The SODAR has no moving parts, enhancing the reliability of the experiment throughout the Antarctic winter.

- The SODAR is a remote sensor and therefore does not affect the turbulence profile. Other turbulence sensors such as towers and balloons have been criticised for interfering with the wind profile. The physical presence of these sensors can induce an extra local turbulence that is measured by the device and, therefore, overestimate the turbulence level and perturb the wind profile. A SODAR uses sound waves and thus does not suffer the same problem. The sensing is done without interfering with the wind conditions and is fast enough to ensure that the measurements at each altitude are quasi-simultaneous.

- The SODAR is capable of measuring the 3-D profile of the wind velocities and therefore gives the opportunity to examine the relation between turbulence and the wind conditions.

The SODAR used for this experiment is a monostatic SODAR model PA1 manufactured by Remtech. Its antenna, placed on top of the AASTO, emits a series of 0.20 second long pulses at 5 different frequencies. The antenna then switches itself 


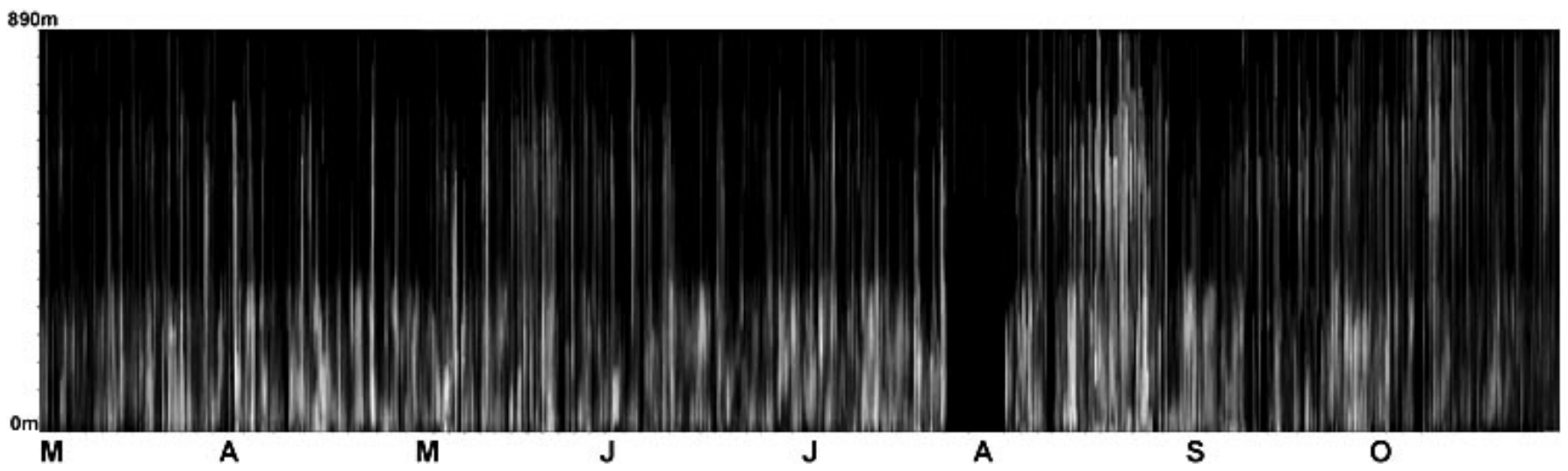

Fig. 1. "Facsimile" plot of the turbulence profile between 2000 March and November (the letters on the $x$-axis mark the beginning of the month). The $y$-axis shows altitude from 0 to 890 metres while the brightness intensity shows the turbulence intensity. The turbulence intensity drops sharply between 200 and 400 metres, defining the boundary layer height.

to receiving mode and records the echo pattern. This routine is repeated for an averaging period of $20 \mathrm{~min}$, including sequences without emission that are used to calculate the background noise. After the averaging, the SODAR returns the echo strength which is proportional to $C_{T}^{2}$. The wind speed is derived from the Doppler shift of the returned echoes; the vertical component from the vertical beam and the horizontal components from the beams slanted at $45^{\circ}$ to the vertical. As previous studies have shown that the majority of the turbulence was concentrated in the lowest $300 \mathrm{~m}$ of the atmosphere, we configured the SODAR to make measurements from $20 \mathrm{~m}$ to $890 \mathrm{~m}$ with $30 \mathrm{~m}$ increments. In order to verify the accuracy of the SODAR, wind data were compared to simultaneous balloon launched meteorology measurements. These data showed good agreement over the whole altitude range covered by the SODAR.

Data were accumulated almost continuously every halfhour from 2000 February 13 to 2000 November 11. After elimination of poor signal-to-noise data, there are a total of 8644 sets of measurements. As the SODAR is not able to calculate the temperature and atmospheric pressure as function of height, the data from daily weather balloon launches were used to convert $C_{T}^{2}$ to $C_{N}^{2}$ (see Eq. (7)). A linear interpolation was used for altitudes where temperature and pressure data were not available. We also made the assumption that the temperature and pressure temporal fluctuations are small over a period of 24 hours because of the continuous night time.

\subsection{Calibration}

The echo strength given by the SODAR is proportional to the temperature fluctuation constant. The conversion to absolute values of $C_{T}^{2}$ and $C_{N}^{2}$ is only necessary for the calculation of the seeing and other astronomical parameters; determining the turbulence profile itself does not require an absolute calibration.

To convert our data to approximate values of $C_{T}^{2}$, we compared our $C_{N}^{2}$ profiles with Marks' microthermal balloon measurements (Marks et al. 1999). These measurements, made in the winter of 1995 , include a total of 15 balloon launches that recorded the temperature difference between two microthermal sensors as a function of altitude. The difficulty and uncertainty of the calibration comes from the non-simultaneity of the SODAR data with the microthermal data. However, the extensive coverage of the winter season allows us to look for similar turbulence profiles to those encountered during the balloon launches. In addition to giving a rough calibration, a comparison between these data allows us to determine the SODAR's sensitivity, ie the lowest $C_{N}^{2}$ value that the SODAR can detect. We assume:

$C_{N}^{2}=A \times C_{N}^{2}(S O D A R)+B$,

where $A$ is the calibration coefficient and $B$ the SODAR minimum sensitivity. The minimum sensitivity is independent of height as the SODAR automatically spends more time on the higher turbulence during the $15 \mathrm{~min}$ integration to compensate for the loss signal intensity induced in the beam spread. The method used is the following: for each of the balloon flights, an average of all the SODAR profiles displaying a correlation with the microthermal measured turbulence above $90 \%$ was calculated. This gave 15 linear regressions which in turn were averaged. Finally the calibration obtained was applied to the entire data set. The systematic errors involved with the calibration are difficult to assess, but include the microthermal errors and the SODAR errors. There are also statistical errors induced in the calibration. The errors presented in the rest of this paper are the internal standard deviations of the data set, and so do not include systematic effects.

\section{Turbulence results}

\subsection{Turbulence and boundary layer evolution}

From here on, we define the boundary layer altitude as being the height above which the turbulence amplitude is below the SODAR threshold of detection. Calibration has shown that this threshold is two orders of magnitude lower that the average turbulence.

The turbulence measurements taken from the 8600 sets of data are displayed in Fig. 1. The boundary layer altitude is of primary importance when studying a potential telescope site in Antarctica. During the period of our measurements, the turbulence is concentrated on average below $270 \mathrm{~m}$. It is notable 
that this is higher than the $220 \mathrm{~m}$ estimate by Marks et al. (1999), who accumulated data from late June to mid August, the time of year giving the best seeing conditions of the site. This is in the peak of winter (June and July), when there is the largest temperature gradient and the most concentrated boundary layer, including a long period of stable atmosphere ( 9 days of 2000 July have a derived seeing at the surface of below 0.2 arcsec). Early and late winter conditions are, however, more unstable. Periods of low altitude boundary layer are mixed with periods of extended boundary layer and periods having dual layers. Figure 2 shows the frequency of occurrence of boundary layer altitudes.

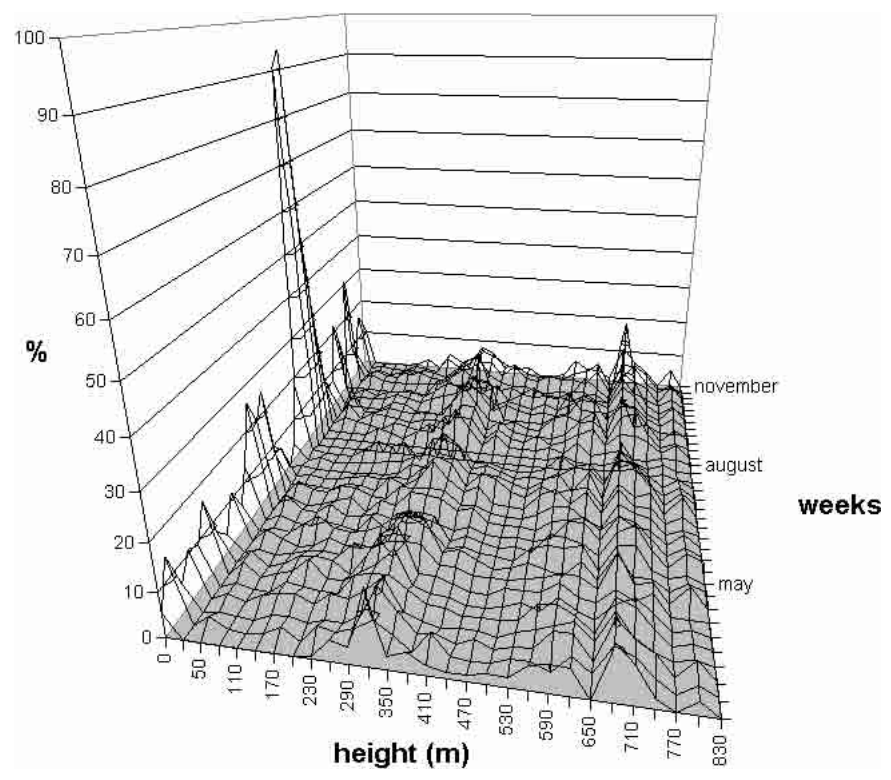

Fig. 2. Frequency of occurrence of the boundary layer altitude. The data are binned into weekly average (first horizontal axis) as a function of height (second horizontal axis) and the result expressed in relative percentage (vertical axis).

The boundary layer height often falls into one of the three bins. The first bin includes the first 30 metres of the atmosphere. It corresponds to a virtually turbulence-free atmosphere, which is mainly present between mid June and the beginning of August. Through out the year, these periods of stable atmosphere correspond to $18 \%$ of the time sampled. The second bin, centred at $260 \mathrm{~m}$, represents the most common boundary layer altitude with an occurrence of $21 \%$. This kind of boundary layer is evenly distributed with time and is a good indication of the expected boundary layer altitude. The third and last bin occurs at $690 \mathrm{~m}$ (present in $17 \%$ of the data). It corresponds to a secondary, usually less intense, drop of the turbulence intensity. This type of boundary layer is more frequent in the weeks surrounding the sunset and the sunrise and is absent from the middle of winter. These three types of turbulence behaviour will be further examined in conjunction with meteorological parameters in the next section.

The evolution of the boundary layer height during the year shows a seasonal trend. It drops continuously after sunset, reaching a minimum in July. It then rises again closer to

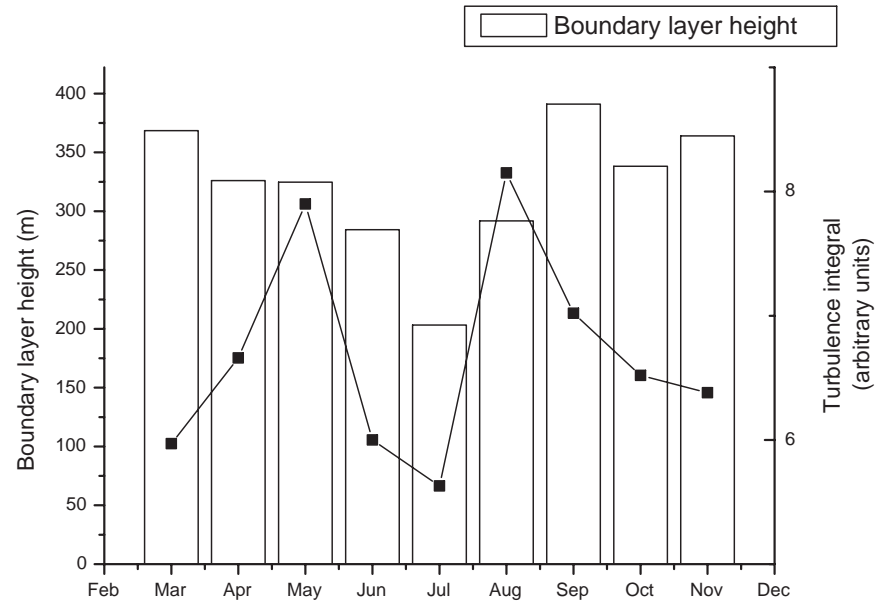

Fig. 3. Monthly distribution of the boundary layer height (columns) and of the total turbulence (line). The boundary layer height evolves according to the seasons, decreasing in altitude towards the peak of winter and increasing again towards sunrise. The total amount of turbulence, on the other hand, does not follow this trend.

summer and sunrise (see Fig. 3). This effect is due to strengthening of the temperature inversion in winter because of the lack of radiative heating. The total amount of turbulence does not, however, follow a similar pattern. While the turbulence is still a minimum in July, there is not a direct correlation between the height of the boundary layer and the total amount of turbulence in the atmosphere.

\subsection{Meteorological parameters}

The boundary layer evolution throughout the year is dependent on two parameters. The first one is the temperature gradient, around which Kolmogorov's theory of turbulence is built. The second parameter is the wind speed. The katabatic winds generated on the high plateau of Antarctica gain speed as they descend across the topographic contours of the continent. The South Pole station is at a relatively high altitude $(2835 \mathrm{~m})$ and the wind speed is much weaker than it is near the coast. It is, however, strong enough to induce turbulence, with ground speeds of $20 \mathrm{~m} \mathrm{~s}^{-1}$ occasionally recorded. Figures 4 and 5 show the close relation between $C_{T}^{2}$ and the horizontal wind speed at corresponding altitudes. The correlation between wind speed and turbulence level has previously been recorded in the coastal regions of Antarctica (Yague et al. 2001) and is confirmed to be present on the plateau by our measurements. The majority of the turbulence is located below the altitude of $300 \mathrm{~m}$ with a secondary and lesser peak at $600 \mathrm{~m}$. The wind profile follows a similar pattern. In fact, the correlation coefficient between $C_{T}^{2}$ and wind speed, using the averages over the whole data as a function of altitude, is a high $91 \%$, suggesting that wind speed measurements alone could be a good predictor of the turbulence profile.

The vertical component of the wind velocity can be further related to turbulence and wind behaviour. Figure 6 shows the average vertical wind speed as a function of height. The boundary layer is characterised by a layer of upward wind $240 \mathrm{~m}$ 


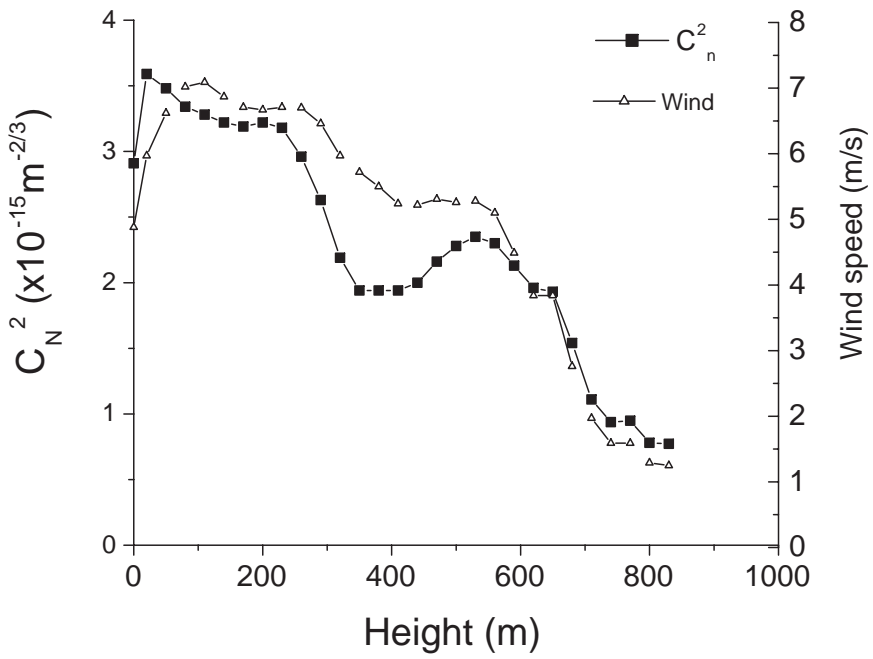

Fig. 4. Turbulence (square dots) and horizontal wind speed (averaged over the whole data set). Both curves display drops at $300 \mathrm{~m}$ and $600 \mathrm{~m}$, representing the two most common boundary layer altitudes.

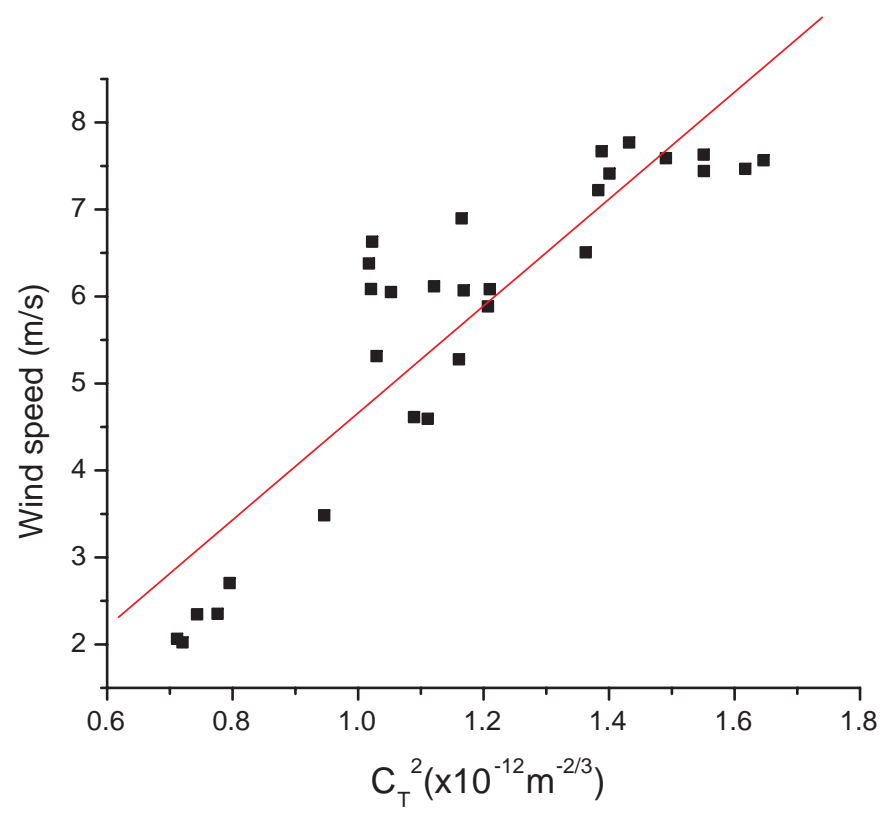

Fig. 5. Correlation between average horizontal wind speed and temperature fluctuation constant. Each point represents an average at a particular height (similar to Fig. 4). Also displayed is the line of best fit.

thick and corresponding to the altitude range of high turbulence. Once again, a correlation analysis shows that the height of the boundary layer and the height of the wind inversion (height at which the vertical component of the wind speed reverses direction) strongly matched, with a correlation coefficient of $92 \%$.

As previously noted, each turbulence profile can be assigned to one of three categories which account for $56 \%$ of the data observed. The remaining $44 \%$ corresponding to states intermediate between the three main states. The first type of turbulence profile (type 1) that is encountered in $18 \%$ of the data is characterised by a thin, ground level, turbulence layer followed by a perfectly stable atmosphere above $100 \mathrm{~m}$. The most

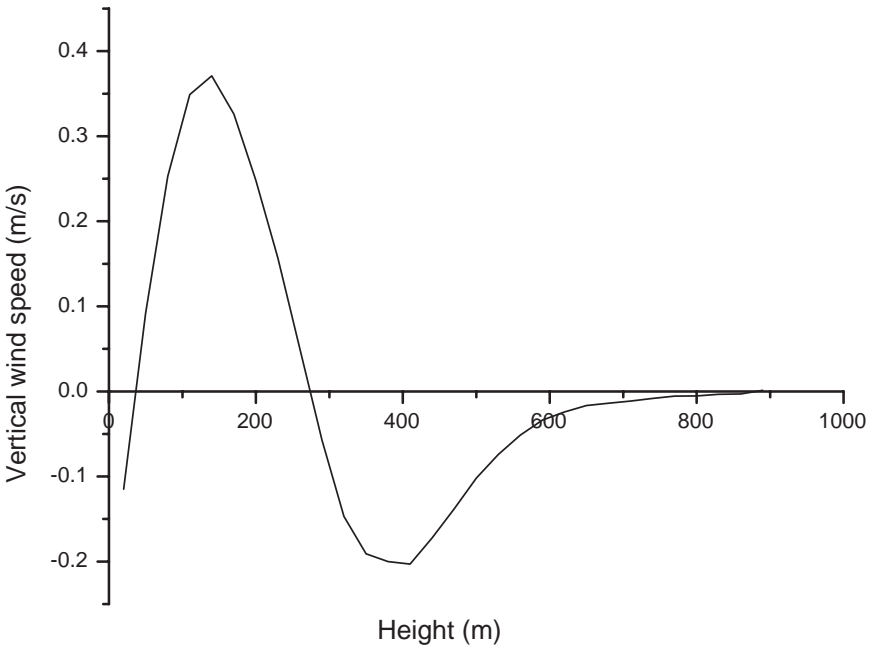

Fig. 6. Average vertical component of the wind speed expressed as a function of altitude. Positive values are upward. They are located over an altitude range similar to the peak turbulence layer.

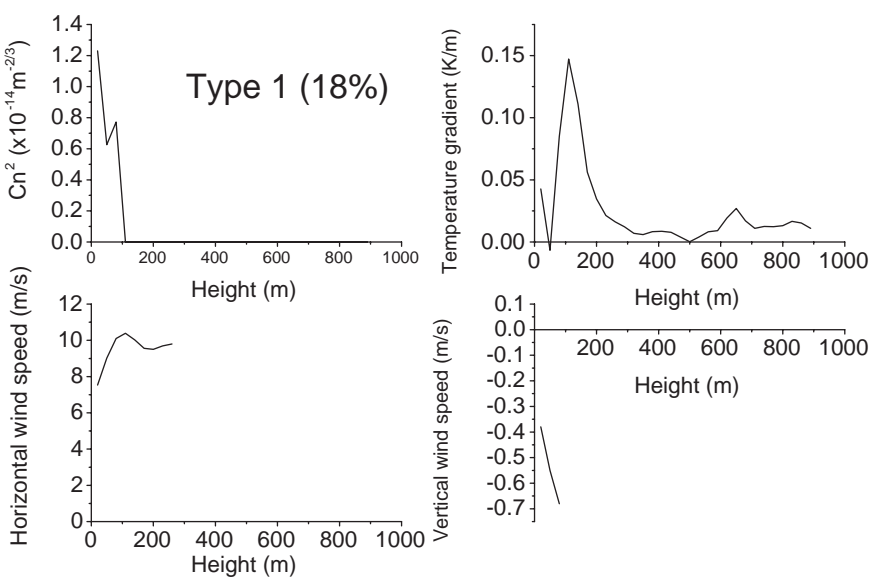

Fig. 7. Typical profiles of type 1 turbulence: semi-stable atmosphere, ground level turbulence. The plot was truncated when the turbulence became too small to be measured.

common type of profile (type 2) occurs $21 \%$ of the time and corresponds to a thick boundary layer occupying the lower $300 \mathrm{~m}$ of the atmosphere. The last type of profile (type 3) is a more extended boundary layer showing a secondary and lower peak of turbulence at around $600 \mathrm{~m}$ and is visible in $17 \%$ of the data. Figures 7-9 show an example of the three types of turbulence and wind profiles recorded by the SODAR as well as the temperature gradient measured on the same day.

The relationship between turbulence and horizontal wind speed described earlier is independent of the type of profile taken by the turbulence. When the turbulence is located in a concentrated altitude boundary, the winds also reach large velocities in a similar pattern. For an extended turbulence like type 3 , the wind profile also becomes more complex. The correspondence between upward winds and turbulence is followed closely for turbulence of type 2 and 3 , where the peak turbulence corresponds to the peak wind speed. For a type 1 turbulence however, the vertical wind speed remains negative. The ground level turbulence is therefore not associated with 

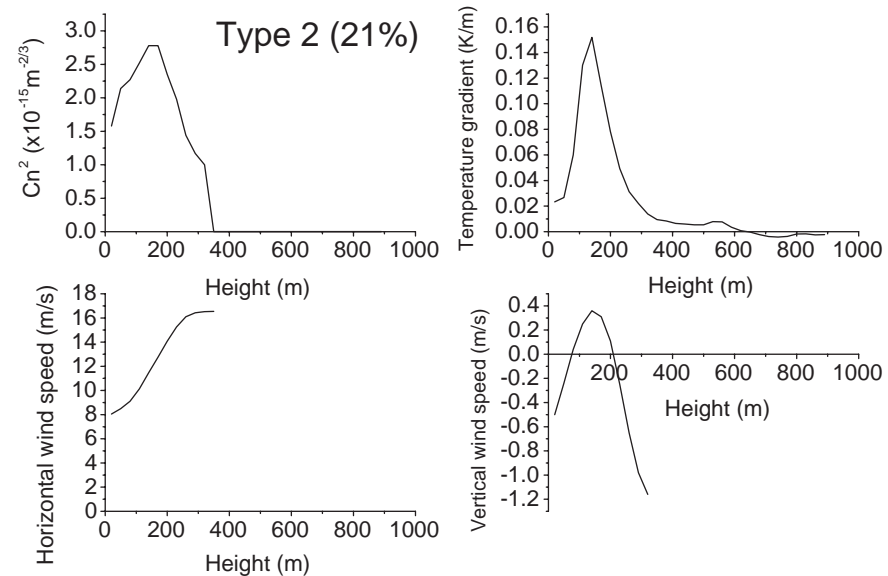

Fig. 8. Type 2 turbulence: concentrated boundary layer. Plots are as in Fig. 7.

a change of wind direction, as it is the case with higher altitude turbulence. This observation implies that the turbulence is generated at ground level $(<100 \mathrm{~m})$ when the winds blow uniformly downward but can be "carried" to higher altitudes as the wind changes direction.

\section{Seeing}

Having described the turbulence profile under $890 \mathrm{~m}$, we can now assess the seeing quality of the site. Since the seeing depends on the integration of $C_{N}^{2}$ over the whole atmosphere, it is necessary to estimate the amount of turbulence above the measurable range. In the absence of a jet stream, there is no reason to expect major turbulence above the boundary layer. To confirm this assumption, we computed the Richardson number, $R_{i}$ as a function of altitude for every day of observation. As described in Marks et al. (1999), the criterion for turbulence is:

$R_{i}=\frac{g}{\theta} \frac{(\partial \theta / \partial h)}{(\partial v / \partial h)^{2}}<\frac{1}{4}$

where $g$ is the gravitational constant, $v$ the horizontal wind speed and $\theta$ the temperature. $v$ and $\theta$ were obtained from balloon measurements. Using this description, $91 \%$ of the time, no turbulence is present above $890 \mathrm{~m}$. The evaluation of the seeing and other relevant atmospheric parameters is therefore an estimate applicable to these $91 \%$ of the data. The statistics are summarised in Table 1 and in Fig. 10. The seeing contribution arising above $300 \mathrm{~m}$ is also displayed in order to emphasise the influence of the boundary layer. In fact, the average seeing could be decreased from $1.7^{\prime \prime}$ to $0.37^{\prime \prime}$ by observing at this altitude.

The values are in close agreement with seeing measurements inferred at the South Pole using the microthermal measurements done by Marks et al. (1999), though the absolute calibration is of course directly from the Marks et al. data. While the SODAR is unable to measure the seeing contribution above $890 \mathrm{~m}$, the analysis of the data using the Richardson number profile would suggest that the free atmosphere contribution should be close to zero. The microthermal measurements of Marks et al. suggest that this contribution is of the order
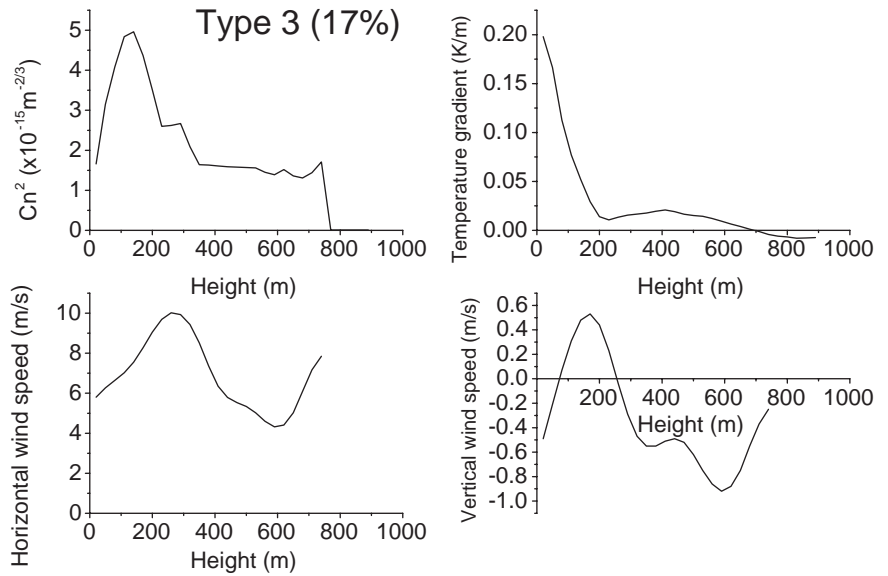

Fig. 9. Type 3 turbulence: extended, 2 component boundary layer. Plots are as in Fig. 7.

of $0.3^{\prime \prime}$. This additional turbulence, which does not add linearly to our measurements (see Eq. (8)), would only add $0.05^{\prime \prime}$ to the average seeing measured by the SODAR. The SODAR seeing data are also consistent with the DIMM measurements of Loewenstein et al. (1998), taken from a $12 \mathrm{~m}$ tower. The results from the three instruments are summarised in Table 2.

\subsection{A telescope on a tower}

Using Eq. (8), and varying the lower limit of the integral, it is possible to estimate the seeing that would be observed with a telescope at a given altitude $h$. Figure 11 shows this evolution calculated from monthly averages, and clearly shows that the seeing drops to an undetectable range. The overall behaviour of these profiles is similar at all times, but the months of winter show sharper drops with altitude as well as lower median values. It is important to note that the lower limit of the seeing reached in all the profiles is fairly qualitative as they usually attain the limit of measurability of the SODAR. The overall seeing distribution is described by Fig. 10 and is in good agreement with seeing measurements made with microthermal sensors and previously discussed by Marks et al. (1996).

\subsection{Other astronomical parameters}

While the ground level seeing conditions of the South Pole do not match those offered by the best Chilean and Hawaiian sites (Fuchs 1995 and Roddier et al. 1990), the unique turbulence profile typical of Antarctica is ideal for image correction schemes such as adaptive optics. As given by Roddier (1981), the two parameters relevant to the conditions of correction are the isoplanatic angle and the coherence time, with variations between adaptive optics (AO) and speckle interferometry (SI):

\section{Isoplanatic angle:}

$\theta_{\mathrm{AO}}=0.31 r_{0}\left(\frac{\int_{0}^{\infty} h^{5 / 3} C_{N}^{2}(h) \mathrm{d} h}{\int_{0}^{\infty} C_{N}^{2}(h) \mathrm{d} h}\right)^{-3 / 5}$, 
Table 1. Summary of the seeing measurements averaged over the whole data set.

\begin{tabular}{lllll}
\hline \hline Measurement & Mean & Std. Dev. & Median & Best 25\% \\
\hline Total Seeing (") & 1.73 & 1.07 & 1.59 & 1.17 \\
Seeing above $300 \mathrm{~m}$ & 0.61 & - & 0.37 & Below detection range \\
Boundary layer height & 267 & 280 & 204 & 70 \\
\hline
\end{tabular}

Table 2. Comparison of the three seeing experiments deployed at the South Pole. All figures are in arcseconds. Note that the absolute calibration of the SODAR data is derived from the microthermal data.

\begin{tabular}{lllllll}
\hline \hline Instrument & Mean & Std. Dev. & Median & Best 25\% & Best & Worst \\
\hline SODAR (This work) & 1.73 & 1.07 & 1.59 & 1.17 & - & 8.11 \\
Microthermals (Marks et al. 1999) & 1.86 & 0.75 & 1.6 & 1.0 & 0.8 & 3.1 \\
H-DIMM (Loewenstein et al. 1998) & 1.53 & - & 1.64 & - & 0.6 & 6.2 \\
\hline
\end{tabular}

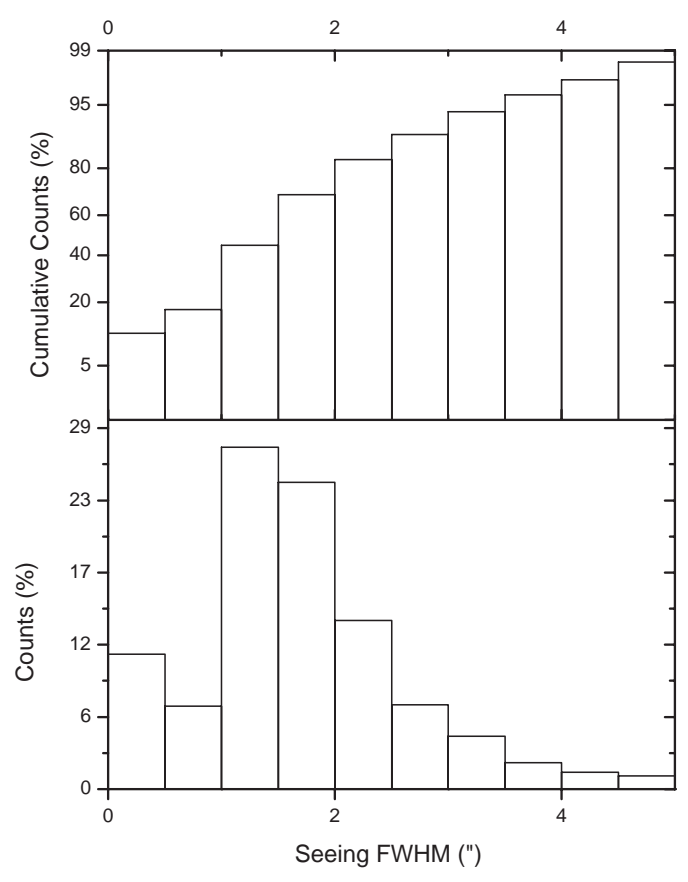

Fig. 10. Seeing cumulative distribution and distribution (expressed as percentages) for the ensemble of the data taken between 2000 March and November at ice level.

$\theta_{\mathrm{SI}}=0.36 r_{0}\left(\frac{\int_{0}^{\infty} h^{2} C_{N}^{2}(h) \mathrm{d} h}{\int_{0}^{\infty} C_{N}^{2}(h) \mathrm{d} h}-\left(\frac{\int_{0}^{\infty} h^{2} C_{N}^{2}(h) \mathrm{d} h}{\int_{0}^{\infty} C_{N}^{2}(h) \mathrm{d} h}\right)^{2}\right)^{-1 / 2}$,

\section{Coherence time:}

$\tau_{\mathrm{AO}}=0.31 r_{0}\left(\frac{\int_{0}^{\infty} v^{5 / 3} C_{N}^{2}(h) \mathrm{d} h}{\int_{0}^{\infty} C_{N}^{2}(h) \mathrm{d} h}\right)^{-3 / 5}$,

$\tau_{\mathrm{SI}}=0.36 r_{0}\left(\frac{\int_{0}^{\infty} v^{2} C_{N}^{2}(h) \mathrm{d} h}{\int_{0}^{\infty} C_{N}^{2}(h) \mathrm{d} h}-\left(\frac{\int_{0}^{\infty} v^{2} C_{N}^{2}(h) \mathrm{d} h}{\int_{0}^{\infty} C_{N}^{2}(h) \mathrm{d} h}\right)^{2}\right)^{-1 / 2}$.

The isoplanatic angle can be regarded as the part of the sky inside which all parts of the image distort with the same phase. Adaptive optics relies on a guide star in order to compensate for the image variation. This reference star must belong to the same turbulence field in order to correct the seeing properly (Fusco et al. 2000). The larger the isoplanatic angle, the larger the chance of finding a bright star in phase with the observed object. The isoplanatic patch depends critically on the altitude distribution of the turbulence. The lower the turbulent cell, the larger the angle it will occupy in the sky. In Antarctica, the light passes through only one layer of turbulence concentrated at low altitude, and conditions for adaptive optics correction are therefore considerably superior to other sites.

The coherence time is the time equivalent of the isoplanatic patch. The amount of time in which a cell of turbulence remains in the field of view will determine the length of time over which the correction is accurate. Since turbulent cells are moved around by the wind, it is the wind speed distribution that will dictate the time of coherence. Again, the South Pole wind conditions are advantageous as they are only substantial within the boundary layer.

As the isoplanatic angle has a strong dependence on height, the SODAR data were extended by adopting the average microthermal data between $890 \mathrm{~m}$ and $24 \mathrm{~km}$. The wind profile was also extended to $24 \mathrm{~km}$ assuming a $2 \mathrm{~m} \mathrm{~s}^{-1}$ wind speed above $890 \mathrm{~m}$ (typical winter wind speed as recorded by the meteorological team). Table 3 summarises the values for the isoplanatic angle and coherence time and compares them with the sites of Cerro Paranal, in Chile and La Palma, in the Canary Island. Boundary layer corrected figures (above $300 \mathrm{~m}$ ) are also listed to emphasise the potential gain associated with the rise of the telescope.

Two other parameters, that are not the subject of this paper, but potentially derivable from the SODAR measurement, benefit from the South Pole turbulence distribution. Interferometry suffers from phase errors that again dependent on the altitude of the turbulence. The mean square error for an astrometric measurement with a dual-beam, differential interferometer is, as described in Lloyd et al. (2002):

$\sigma_{\delta}^{2}=5.25 B^{-4 / 3} \theta^{2} \int h^{2} C_{N}^{2}(h)(V t)^{-1} \mathrm{~d} h$

where $B$ is the baseline of the interferometer, $\theta$ is the angular separation of the celestial objects, $V$ is the wind speed as a function of height and $t$ is the total integration time. The $h^{2}$ factor in this equation shows the advantage that low high-altitude 
Table 3. Summary and comparison of the South Pole correction parameters at $500 \mathrm{~nm}$ with two temperate sites.

\begin{tabular}{lllll}
\hline \hline Site & $\theta_{\mathrm{AO}}\left({ }^{\prime \prime}\right)$ & $\theta_{\mathrm{SI}}\left({ }^{\prime \prime}\right)$ & $\tau_{\mathrm{AO}}(\mathrm{ms})$ & $\tau_{\mathrm{SI}}(\mathrm{ms})$ \\
\hline South Pole (total) & 3.3 & 2.8 & 2.9 & 13 \\
South Pole (B.L correction) & 63 & 193 & 3 & 35.5 \\
Cerro Paranal (Fuchs 1995) & 1.45 & 1.88 & - & - \\
La Palma (Vernin et al. 1994) & 1.3 & 2.2 & 7 & 13 \\
\hline
\end{tabular}

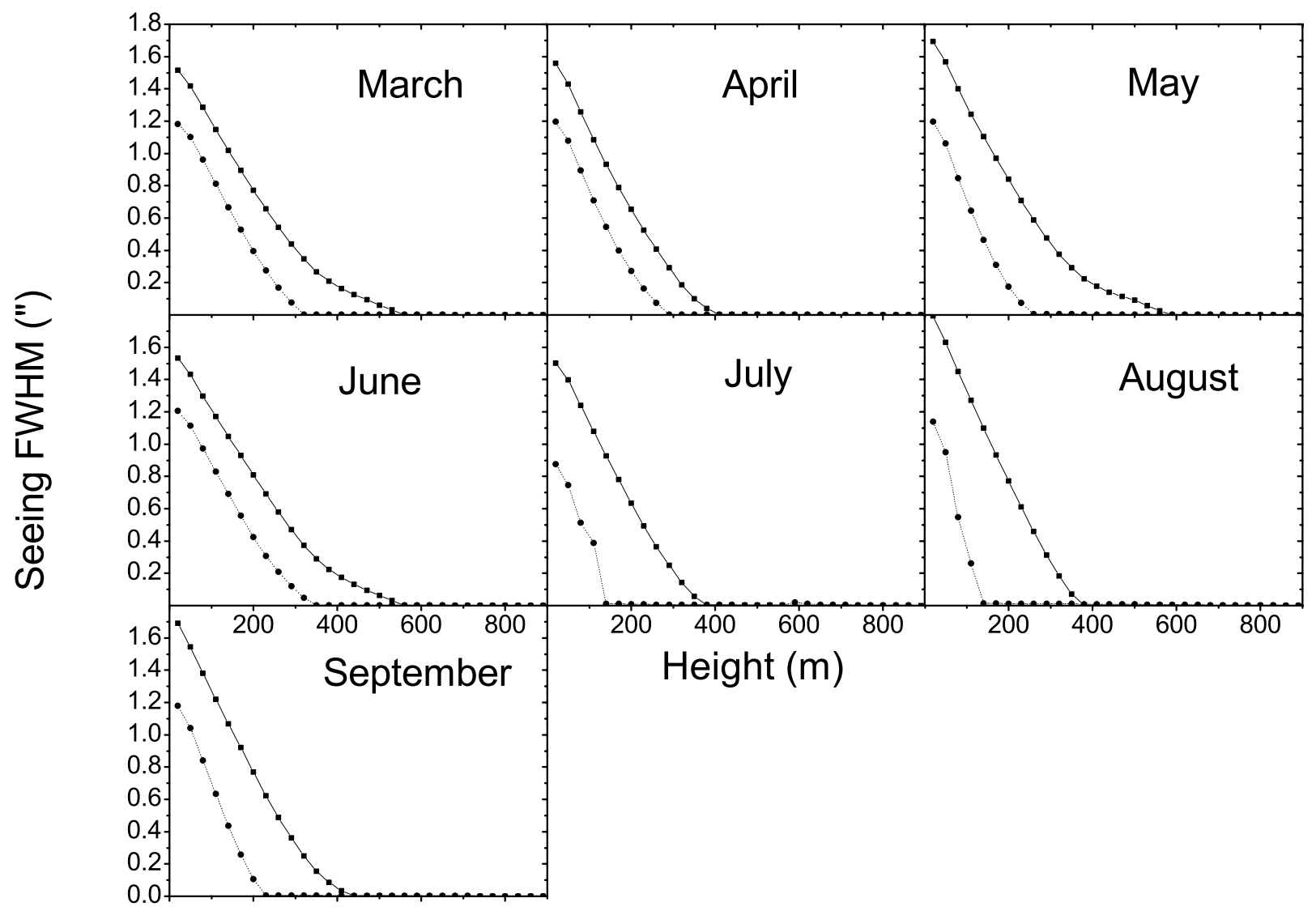

Fig. 11. Monthly averages of the seeing as a function of telescope height above the ground during 2000. The mean is represented by the full lines and the median values by the dotted lines.

turbulence can have on astrometric measurements. In Lloyd et al. (2002), a turbulence model adapted from existing data compares the mean square error of South Pole with Mauna Kea. The results show that the South Pole mean square error is a factor of 15 better than in Hawaii, where the turbulence is mostly located a few thousand metres above the ground.

Flux variation measurements also take advantage of the low levels of high-altitude turbulence of Antarctica. Observations of variable stars and solar pulsations (Fossat et al. 1981) are limited by the change of flux created by our own atmosphere. These changes are expressed by the scintillation index:

$\sigma_{I}^{2}=19.12 \lambda^{-7 / 6} \int h^{5 / 6} C_{N}^{2}(h) \mathrm{d} h$

and correspond to the variation of the intensity of the studied object. The altitude dependence of the scintillation is not as critical as it is for the astrometric error. It is however significant enough to make the scintillation at the South Pole smaller than at Cerro Paranal by a factor of 2, using the microthermal data of Marks et al. (1999).

\section{Interpretation and future work}

The relationships we have observed between wind speeds and the directly measured turbulence intensity, as summarised in Table 4, help us understand the evolution of the turbulence profile throughout the year. During winter period the boundary layer is the most well defined. Closer to day time, the more the turbulence diffuses out to higher altitude. In winter, the ice radiates into space, cooling itself and the air close to the ground. This creates a strong temperature inversion. In summer, the sun heats the ice and the air close to it, creating a much smaller (and negative) temperature gradient with lower associated turbulence.

Figure 12 shows the strong correlation between the height of this wind inversion and the altitude of the mixing height 
Table 4. Summary of the turbulence and wind correlations.

\begin{tabular}{lccc}
\hline \hline & \multicolumn{3}{c}{$\begin{array}{c}\text { Regression line } \\
\end{array}$} \\
Relationship $(y, x)$ & $R$ & $a$ & $b$ \\
\hline Horizontal wind & 0.91 & 6.15 & -148 \\
speed and turbulence. ${ }^{(1)}$ & $\left(\mathrm{m}^{5 / 3} \mathrm{~s}^{-1}\right)$ & $\left(\mathrm{m} \mathrm{s}^{-1}\right)$ \\
\hline $\begin{array}{l}\text { Positive, vertical wind speed } \\
\text { range and turbulence range }\end{array}$ & 0.92 & 1.14 & 56 \\
\hline $\begin{array}{l}\text { Mixing height and } \\
\text { wind inversion size. }\end{array}$ & & & $(\mathrm{m})$ \\
\hline
\end{tabular}

$R$ is the correlation coefficient between the two parameters, $a$ and $b$ are the linear regression terms. In ${ }^{(1)}$, the average turbulence and average wind speed were used. The correlation was calculated using points at the same altitudes and is shown in Fig 5 . In ${ }^{(2)}$, the height of the boundary layer was compared to the size of the positive vertical speed range for individual sets of data. $\operatorname{In}^{(3)}$, monthly averages of the mixing height and wind inversion size were compared and shown in Fig. 12.

(altitude at which the temperature starts decreasing). The second effect of the radiative heating is the extension of the warmer layer of air close to the ground to higher altitudes. The elevation of the mixing height therefore also raises the size of the boundary layer (Neff 1981). Often, this elevation gives birth to a secondary peak near the mixing height. While this effect is unexpected it has been observed before at the coastal base of Halley (Rees et al. 1988). While this secondary peak limits the effectiveness of using a tower to increase the seeing quality, it occurs sufficiently infrequently that elevating the telescope is justified. As every ten metres has a large impact on the improvement of the seeing, the maximum seeing limitations of an adaptive optics system will dictate the altitude at which telescopes must be built.

The dependence of the turbulence on the wind profile will help us in the future to predict the turbulence characteristics at any site on the Antarctic continent as the meteorological parameters become available. In turn, the turbulence distribution determines the level of adaptive optics correction needed from a site. The kind of correction necessary is also expected to be much simpler. This is due to the fact that in temperate sites, the turbulence caused by the jet stream is spread out over multiple layers. The South Pole turbulence is however confined to only one, low altitude boundary layer.

In order to confirm our expectation, our group is jointly working with a team from the Australian National University on a twenty-four aperture DIMM also located at the South Pole. The twenty four apertures of the DIMM will enable us to work out the degree of distortion of the light wave front, which can be compared with the type and altitude of turbulence observed by the SODAR. This work will quantify the level of adaptive optics correction required on the future telescopes built for Antarctica.

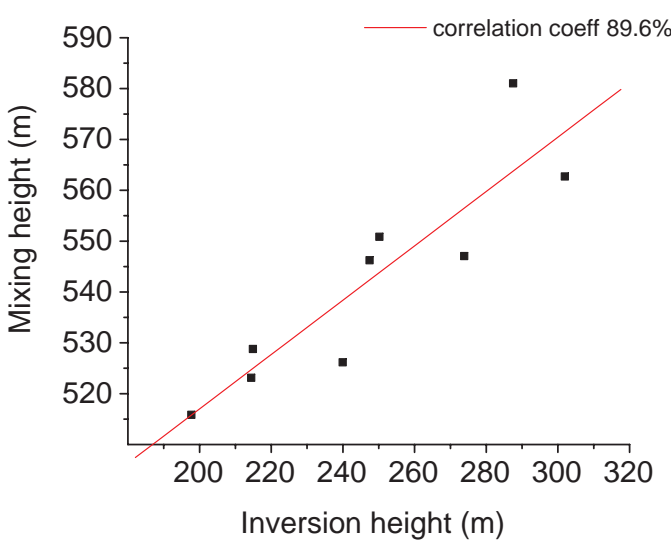

Fig. 12. Plot of the mixing height (altitude at which the temperature starts decreasing) as a function of the wind inversion height. Each point corresponds to a monthly average.

\section{Conclusions}

Our key results can be summarised as follow:

- The winter turbulence above the South Pole is characterised by a single, low altitude component. Autumn and Spring vary between a concentrated and an extended profile of turbulence.

- The turbulence as a function of height follows closely the horizontal wind profile while always sitting below it.

- The turbulence peaks in the zone of vertical wind inversion.

- The average seeing, while poor at ground level (1.73"), improves very quickly within the first $300 \mathrm{~m}$ of the atmosphere $\left(0.61^{\prime \prime}\right)$.

- The extent of the boundary layer might be too high to place a telescope on a high tower, but is concentrated enough that the isoplanatic angle and coherence time are the best ever observed. Through the use of adaptive optics, the South Pole could outperform all other sites so far studied.

Acknowledgements. This research was supported by grants from the Australian Research Council (ARC) and the University of New South Wales (UNSW). Logistic and infrastructure support were supplied by CARA and the NSF. We thank Daniel Marlay and Jessica Dempsey for help in "winterising" the SODAR and installing it at the South Pole. We also would like to thank Marc Sarazin for his advice on the use of a SODAR to investigate the seeing as well as J. Beckers for his valuable comments.

\section{References}

Crescenti, G. H. 1997, Bulletin of the American Meteorologiacal Society, 78 (4), 651

Danilov, S. D., Gur'yanov, A. E., et al. 1992, Meas. Sci. Technol, 3, p. 1001-1007

Fossat, E., Grec, G., \& Pomerantz, M. 1981, Sol. Phys., 74, 59-63

Fried, D. L. 1966, J. Opt. Soc. Am., 56, 1372

Fuchs, A. 1995, Contribution à l'étude de l'apparition de la turbulence optique dans les couches minces. Concept du Scidar généralisé (Ph.D. Thesis), Université de Nice-Sophia Antipolis, France

Fusco, T., Conan, J.-M., Michau, V., Rousset, G., \& Mugnier, L. 2000, SPIE, 4007, 1044 
Hecquet, J., \& Klaus, V. 1989, A\&A, 225, 585

Lloyd, J. P., Oppenheimer, B. R., \& Graham, J. R. 2002, PASA, 19, 3 Loewenstein, R. F., Bero, C., Lloyd, J., et al. 1998, ASP Conf. Ser., 141,296

Neff, W. D. 1981, Ph.D. Thesis, Wave Propagation Laboratory (Boulder Colorado, USA)

Marks, R. D., Vernin, J., Azouit, M., et al. 1996, A\&A, 118, 385

Marks, R. D., Vernin, J., Azouit, M., Manigault, J. F., \& Clevelin, C. 1999, A\&AS, 134, 161

Rees, J. M., \& Mobbs, S. D. 1988, Q. J. R. Meteorol. Soc., 114, 939

Roddier, F. 1981, in Progress in Optics, ed. E. Wolff, 19, 283-377
Roddier, F., Cowie, L., Graves, J. E., et al. 1990, SPIE, 1236, 485

Storey, J. W. V. 1998, Astrophysics From Antarctica, ASP Conf. Ser., 141,313

Storey, J. W. V., Ashley, M. C. B., \& Burton, M. G. 1996, PASA, 13, 35-38

Tatarski, V. I. 1961, Wave propagation in a turbulent medium (New-York: McGraw-Hill)

Vernin, J., \& Muñoz-Tuñóz, C. 1994, A\&A, 284, 311

Yague, C., Maqueda, G., \& Rees, J. M. 2001, Dynamics of Atmospheres and Oceans, 34(2-4), 205 